\title{
Diagnostic Value of Endoscopic Retrograde Cholangiopancreatography and Therapeutic Value of Endoscopic Sphincterotomy in Dogs With Suspected Hepatobiliary Disorders
}

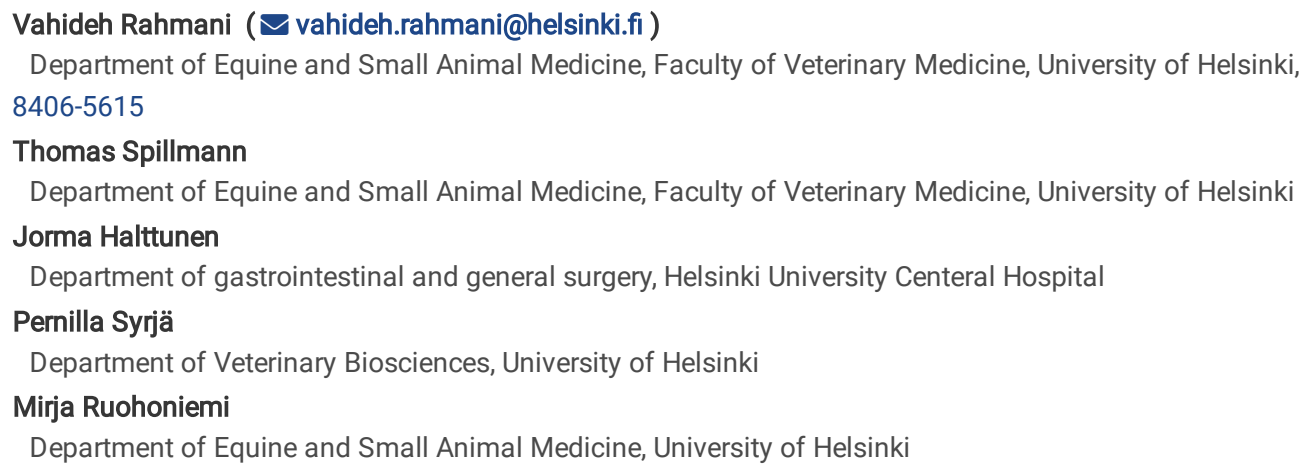




\section{Abstract}

Background: Conventional diagnostic methods have some limitations in diagnosing specific causes of canine hepatobiliary disorders. In the evaluation of the hepatobiliary system in dogs, ultrasonography (US) is the first imaging method of choice. Nonetheless, endoscopic retrograde cholangiopancreatography (ERCP) has also been proven to be a practicable technique for evaluating canine hepatobiliary (endoscopic retrograde cholangiography, ERC) and pancreatic duct (endoscopic retrograde pancreatography, ERP) disorders, providing additional therapeutic options by sphincterotomy (EST). To date, the efficacy and safety of diagnostic and therapeutic ERCP has not been evaluated in veterinary medicine literature. The present study sought to report complications and outcomes of dogs undergoing ERCP and EST, and to assess the usefulness of diagnostic ERCP by comparing the findings of US, ERCP and histopathological findings in liver and pancreas.

Results: This retrospective case series comprises data collected from 15 dogs that underwent successful ERC/ERCP. Nine dogs underwent EST following ERC US and ERC were most in agreement when assessing the common bile duct. In case of disagreement between the modalities, the ERC findings of the ductal structures were in line with the available pathology findings more often than the US findings, whereas the opposite was noted for the gallbladder. The technical success rates were $88.2 \%$ for ERC, $66.7 \%$ for ERP, and $81.8 \%$ for EST, with no major complications during or immediately after the procedure. Immediate bile flow after EST was recorded in 7/9 dogs but only four showed coinciding clinical and laboratory improvement and four dogs were euthanized within one to six days after EST.

Conclusions: US remains a valuable initial diagnostic imaging method for hepatobiliary disorders and allows good assessment of the gallbladder. ERC can serve as a complementary procedure for diagnostic assessment of the hepatobiliary duct disorders. However, in order to improve the outcomes of EST, careful selection of patients for the procedure would require more advanced diagnostic imaging of the hepatobiliary area.

\section{Background}

Conventional diagnostic procedures including history, physical examination and liver biochemical profile have some limitations in diagnosing specific causes of disorders of the canine hepatobiliary system and exocrine pancreas. In cases of hepatobiliary diseases, organ biopsies are required for histologic verification, but liver biopsy is an inherently invasive procedure that carries some complications [1, 2]. Regarding chronic pancreatic diseases, the diagnosis often remains tentative since pancreatic biopsies are rarely taken for histologic confirmation and differentiation [3].

Diagnostic imaging of pancreaticobiliary diseases includes various techniques that are selected based on clinical signs, the diagnostic information sought, the technique's invasiveness and the therapeutic needs of each individual case. Ultrasonography (US) is the most commonly used non-invasive imaging modality in the evaluation of the hepatobiliary system and the exocrine pancreas in dogs, offering high sensitivity but often lacking specificity, especially in patients with biliary and pancreatic ductal problems [4]. Endoscopic retrograde cholangiopancreatography (ERCP) has been proven to be an applicable, direct technique for evaluating hepatobiliary and pancreatic disorders both in human and veterinary medicine $[5,6,7,8,9,10]$. It is a minimally invasive technique, which combines endoscopy and fluoroscopy to image the biliary system (endoscopic retrograde cholangiography, ERC) and pancreatic duct (endoscopic retrograde pancreatography, ERP). With the introduction of endoscopic sphincterotomy (EST), therapeutic ERCP was subsequently developed [11]. Compared to US, ERCP requires general anesthesia, and complications such as pancreatitis, cholangitis, cholecystitis, bleeding and duodenal perforation have been reported in people $[12,13,14]$.

A shift towards using ERCP for treatment purposes has emerged in human medicine together with a wider use of magnetic resonance cholangiopancreatography (MRCP) and endoscopic ultrasound [15]. In veterinary medicine, the feasibility of diagnostic ERCP in dogs with and without chronic gastrointestinal disorders [5, 9] and in healthy cats [8] has been demonstrated. In 2015, Berent et al. reported successful endoscopic biliary stenting in 5/7 healthy dogs and in 1/2 dogs with extrahepatic bile duct obstruction [10]. However, the efficacy and safety of diagnostic and therapeutic ERCP has not been evaluated in the veterinary literature to date.

The primary aim of the present retrospective study was to characterize clinical, laboratory, ultrasonographic and hepatopancreatic histopathological findings, and to report complications and outcome of dogs undergoing ERC/ERCP for diagnosis and EST for treatment. The secondary aim was to compare ultrasonographic and ERC/ERCP findings for assessing the usefulness of diagnostic ERC/ERCP in comparison to abdominal ultrasound.

\section{Results \\ Dogs}

Altogether 17 dogs underwent ERC or ERC with concurrent ERP between November 2007 and March 2019. The major papilla was identified in 17 and the minor papilla in 9 of the 17 dogs. ERC without the pancreatic component was performed successfully on 15 of the 17 dogs (88.2\%) and concurrent ERP on 6 of 9 dogs $(66.7 \%)$. Cannulation of the major papilla had failed in one small-sized dog $(4.7 \mathrm{~kg})$ and another dog had a mass at the major papilla, which prevented the insertion of the catheter. Thus, 15 dogs met the inclusion criteria.

At the time of ERC/ERCP, the dogs had a mean age of $6.8 \pm 2.9$ years and a mean body weight of $17.1 \pm 8 \mathrm{~kg} .0 \mathrm{f}$ the 15 dogs, 8 were male ( 1 neutered) and 7 were female (4 spayed). The included 15 dogs represented 14 breeds (Table 1).

\section{Clinical signs}

Dogs were presented with the following chronic gastrointestinal signs ordered by their frequency of appearance: chronic or recurrent vomiting (14/15), icterus (9/15), chronic or recurrent diarrhea (4/15), recurrent abdominal pain (4/15), recurrent inappetence (4/15), weight loss (3/15), and borborygmus (1/15). 
Clinical signs appeared alone or together (outlined in Table 1).

\section{Laboratory parameters before ERC/ERCP}

A complete serum biochemical panel before ERC/ERCP was available for all dogs. Deviations from the reference range were mainly seen for the serum parameters of the liver (Table 1). Hyperbilirubinemia was present in $9 / 15$ dogs (60\%) with median serum bilirubin of $176.6 \mu \mathrm{mol} / \mathrm{I}$ (range $14-786.4 \mu \mathrm{mol} / \mathrm{I})$. All icteric dogs (Dogs 1-9) had concurrent elevated activities of alkaline phosphatase (ALP) and alanine aminotransferase (ALT). Overall, serum activity of ALP and ALT was elevated in 10/15 dogs and canine pancreatic lipase (CPL) was elevated in five dogs $(534.8 \pm 322.4 \mu \mathrm{g} / \mathrm{l})$.

\section{Abdominal ultrasonography}

The findings of abdominal US of the liver parenchyma, the biliary system and the pancreas were grouped in icteric and non-icteric dogs and are summarized in Table 2. The appearance of the major papilla was recorded only for five icteric and one non-icteric dogs. The pancreatic duct was not mentioned in any of the reports.

All icteric dogs showed changes in the echogenicity of the liver parenchyma. In one dog (Dog 6), the liver appeared heterogenous with multiple hypo- and hyperechoic nodules. In three dogs, the major papilla was prominent. Gallbladder abnormalities were seen in $6 / 9$ dogs with mild-to-moderate echogenic debris, associated with hyperechoic content attached to the gallbladder wall in one dog (Dog 3). The Common bile duct (CBD) was dilated in five dogs. There was no mention of the extrahepatic ducts, but two dilated intrahepatic ducts were reported in one dog (Dog 5). The echogenicity of the pancreas showed abnormal changes in $6 / 9$ dogs.

All six non-icteric dogs had normal hepatic parenchyma in US and a prominent major papilla was reported in one dog (Dog 14). Two dogs had mild-tomoderate gallbladder debris, one dog a mucocele (Dog 12) and one dog (Dog 14) showed a concurrent hyperechoic focus (40 mm) with acoustic shadowing inside the gallbladder. The CBD was dilated in one dog (Dog 13) (FIG 1). No ultrasonographic findings were reported for the extra- and intrahepatic ducts. The echogenicity of the pancreas showed abnormal changes in $4 / 6$ dogs.

\section{ERC/ERCP findings}

The ERC/ERCP findings of the dogs are reported in Table 2.

Conventional endoscopy of the icteric dogs revealed a prominent major papilla in 3/9 dogs and a prominent minor papilla in one dog (Dog 2). In ERC, the CBD was considered dilated in 4/9 dogs and narrowed in 2/9 dogs. In one dog (Dog 2), the gallbladder and CBD did not fill with contrast (FIG 2). The gallbladder and CBD were not assessable in another dog (Dog 7), since the contrast medium leaked out of the major papilla during the procedure. Four dogs (Dogs $2,5,6$, 7) had dilation of the extrahepatic bile ducts and in one dog (Dog 3) dilated intrahepatic bile ducts were visible (FIG 3). In Dog 4, no contrast reached the intrahepatic bile ducts even after several attempts with repositioning of the catheter. ERP was performed in 4/9 icteric dogs, and all of them showed normal findings of the pancreatic ducts.

Conventional endoscopy of the six non-icteric dogs revealed an abnormal major papilla in three dogs. The minor papilla was endoscopically normal in five dogs and one dog did not have any endoscopic report of the minor papilla. ERC revealed a dilated CBD in one dog (Dog 13) (Fig. 1), and in one dog (Dog 10) the CBD was found to course in a half circle around the duodenum and not straight to the gallbladder (Fig. 4). Extrahepatic ducts were normal in all three nonicteric dogs having reports in ERC. One dog had decreased filling of the intrahepatic duct (Dog 14). Out of the three non-icteric dogs with ERP examination, one dog revealed abnormal course of duodenal branch of the pancreatic duct, going cranial to the diaphragm (Dog 15).

\section{Comparison of ultrasonographic, ERC/ERCP and histopathology findings}

When comparing ultrasonographic and ERC/ERCP findings with each other, and where possible with findings of histopathology of the samples taken at endoscopy for ERC/ERCP, laparoscopy, laparotomy or autopsy (Table 3), the following agreements and disagreements were seen concerning the major papilla, gallbladder, CBD, extrahepatic ducts, intrahepatic ducts and pancreatic duct. Reports of both US and endoscopy for ERC for the major papilla were available for $6 / 15$ dogs. There was full agreement on a normal papilla in one dog (Dog 3) and on a prominent papilla in two dogs (Dogs 6 and 8 ). In three dogs with disagreements between US and endoscopy, no morphologic examination of the papilla was possible for final assessment.

Reports of both modalities for gallbladder were available for 11/15 dogs. There was full agreement on a normal gallbladder in six dogs (Dogs $1,4,9,10,11$, 15). From five dogs with different US and ERC findings, Dogs 2 and 7 showed a distended gallbladder with debris in US but no contrast filling of the gallbladder in ERC. Histopathology in Dog 2 revealed biliary stasis, and in Dog 7 cystic mucinous hyperplasia of the gallbladder mucosa. Dogs 5,13 and 14 had distended gallbladder with debris in US, but normal gallbladder in ERC. Laparotomy of Dog 5 revealed a big and pink gallbladder, which was easily emptied when manually pressed. Histopathology showed necrotizing cholecystitis in Dog 14.

Both US and ERC reports of the CBD were available for all 15 dogs. Agreement on a normal CBD occurred in six dogs (Dogs $4,9,11,12,14,15)$ and on a dilated CBD in four dogs (Dogs 3, 6, 8, 13). From five dogs with disagreement between US and ERC, Dogs 1,5 and 10 had a normal CBD in US but ERC reported dilation in Dog 1, narrowing in Dog 5 and an abnormal course in Dog 10. Histopathology revealed chronic cholangiohepatitis and biliary stasis in Dog 1 and focal granulomatous peritonitis in Dog 10 involving the CBD, pancreas and intestine together with a torturous course of the duct. In Dog 5 , laparotomy showed a dilated CBD with no stricture. Dogs 2 and 7 had a dilated CBD in US, but no contrast filling was visible in ERC. Histopathology showed pancreatic carcinoma with infiltration into the duodenal wall around the CBD (FIG 2), chronic cholangiohepatitis with carcinoma metastasis and biliary stasis in Dog 2 , and chronic cholangiohepatitis in Dog 7.

Extrahepatic bile ducts were not mentioned in any of the US reports. ERC reports for extrahepatic bile ducts were available in 10 dogs (normal in Dogs $1,4,9$, $11,12,14$; dilated in Dogs $2,5,6,7)$. Histopathology in the four dogs with dilated extrahepatic duct in ERC revealed biliary stasis in Dog 2, due to a pancreatic 
carcinoma infiltrating the duodenal wall and compressing the CBD, hepatic lipidosis without lesions targeting the biliary tract in Dog 5, chronic cholecystitis with cystic mucinous hyperplasia of the gallbladder mucosa in Dog 6 , and chronic cholangiohepatitis and cystic mucinous hyperplasia of the gallbladder mucosa in Dog 7. In one dog (Dog 5), two dilated intrahepatic ducts were reported in US. However, ERC of the same dog revealed a $2 \mathrm{~cm}$ long stricture in the CBD starting from the major papilla and dilated extrahepatic ducts were reported. Laparotomy revealed no obvious cause of the suspected CBD stricture. Histopathology of the liver parenchyma biopsies revealed severe lipidosis but no significant lesions in the intrahepatic ducts.

The pancreatic duct was not mentioned in any of the US reports. ERP reports were available for seven dogs (normal in Dogs 2, 3, 4, 9, 11, 14; abnormal course in Dog 15). Autopsy reports of the pancreas were available for Dog 2 directly after ERC and Dog 14 at day 178 after ERC. Histopathology showed metastasizing exocrine pancreas carcinoma in Dog 2 and acute sever multifocal necrotizing pancreatitis in Dog 14.

\section{EST}

EST was performed in 7/9 icteric and 4/6 non-icteric dogs. The indication for EST included one or more of the following ERC findings: dilated CBD (5 dogs), prominent major papilla (3 dogs), stenosis or small major papilla (3 dogs), narrowed CBD (2 dogs), no contrast filling of the CBD ( 2 dogs), and obstruction of the CBD (2 dogs). The procedure was successful in 9/11 dogs (81.8\%), of which seven revealed bile flow immediately after cutting the major papilla, whereas, no bile flow occurred in two (Dogs 2 and 7).

\section{ERC/ERCP and EST complications}

None of the dogs that underwent ERC/ERCP or EST developed procedure-related severe complications or deaths. Minor complications occurred in two dogs. In one (Dog 1), the tip of the endoscope broke while cannulating for EST. However, all parts of the endoscope were removed, and the procedure ended up in a successful diagnostic ERC. In the other (Dog 11), it was difficult to cannulate the major papilla with the sphincterotome at the first attempt of performing EST. Some opening of the papilla was possible, but the procedure was stopped due to strong intestinal peristalsis, development of bleeding and an intramucosal injection of contrast medium. Four weeks later, the EST procedure was repeated and was successful with opening of the major papilla.

\section{Outcome after EST}

Improvement in the clinical status and the laboratory parameters was noted in $1 / 6$ of the icteric dogs (Dog 3 ) and $2 / 3$ of the non-icteric dogs (Dogs 12 and 13). The elevated serum liver parameters returned to the normal level in all these dogs shortly after EST (Fig. 5). In Dog 3, US showed that the diameter of the CBD decreased from $10 \mathrm{~mm}$ to $4 \mathrm{~mm}$ in one day after the procedure. The dog remained clinically unremarkable until lost for follow up 71 days after EST. Dog 12 , with a gallbladder mucocele, had short-term clinical improvement after EST, but episodes of recurrent abdominal pain returned. The dog remained free of clinical signs throughout the follow up when permanently receiving ursodeoxycholic acid. Four months after EST, US showed a normal pancreas and the appearance of the mucocele was unchanged until the last US examination performed 509 days after EST. Long-term follow up of Dog 13 revealed development of septic peritonitis 306 days after EST and the dog was euthanized on owner's request. Since the owner refused an autopsy the cause of septic peritonitis remained open.

Dog 6 showed some clinical improvement after EST, and in the ultrasonographic examination two weeks later the gallbladder, CBD and major papilla were reported as normal. However, although the markedly elevated serum liver enzymes (ALP and ALP) gradually decreased, they were still above normal level two months after EST (FIG 5) and the dog had repeated phases of fever and abdominal pain being responsive to metronidazole and enrofloxacin. Four months after EST, the dog underwent laparoscopy with liver biopsy and bile aspiration. Histopathology showed chronic hepatitis and chronic cholecystitis with hyperplasia of the gallbladder mucosa. Bacterial culture revealed extended spectrum beta-lactamase (ESBL) E. coli in the bile but was negative for the liver tissue sample. The gallbladder was removed surgically 12 days after laparoscopy and the infection with ESBL E.coli was confirmed in bile and feces samples. After the surgery, the dog recovered fully but the bacterial culture of the feces samples remained positive for ESBL E.coli. The dog was last presented 22 months after surgery having no relapse of fever and abdominal pain but still moderately elevated liver enzymes.

No improvement after EST was seen in $4 / 6$ icteric (Dogs 2, 5, 7, 8 ) and in 1/3 non-icteric dogs (Dog 14). The four icteric dogs were euthanized one to six days after the procedure. Dogs 2 and 7 showed no bile flow from the major papilla after EST and no clinical improvement immediately after the procedure, and the owners opted for euthanasia the next day. Autopsy revealed infiltrative exocrine pancreatic carcinoma in the duodenal wall suppressing the CBD in Dog 2 and cholangiohepatitis with cystic mucinous hyperplasia in the mucosa of the enlarged gallbladder in Dog 7. In Dog 8, the gallbladder and CBD remained dilated in US examination and serum bilirubin concentration was elevated for one day after EST. The owner refused surgical procedures and chose euthanasia two days after EST. Local chronic mural enteritis at the major papilla was diagnosed at autopsy. The fourth dog (Dog 5) was euthanized six days after EST because of its poor clinical condition. It was impossible to establish the cause of the CBD stricture since the owner declined autopsy. The non-icteric dog (Dog 14) lacked follow-up reports shortly after EST, however, six months after EST the dog showed acute diarrhea, vomiting and abdominal pain, coupled with laboratory findings of elevated ALP, ALT and cPL values. A hyperechoic pancreas was visible in US. Exploratory laparotomy revealed gross alterations in the pancreas, and the owner opted for euthanasia and autopsy, which revealed severe acute necrotizing pancreatitis and peripancreatic peritonitis. 
Signalment and serum parameters of 15 dogs before undergoing ERC/ ERCP

\begin{tabular}{|c|c|c|c|c|c|c|c|c|}
\hline \multirow[t]{3}{*}{ Case } & \multirow[t]{3}{*}{ Breed } & \multirow[t]{3}{*}{$\begin{array}{l}\text { Age } \\
\text { (Years) }\end{array}$} & \multirow[t]{3}{*}{$\begin{array}{l}\text { Weight } \\
(\mathrm{Kg})\end{array}$} & \multirow[t]{3}{*}{ Clinical signs } & \multicolumn{4}{|c|}{$\begin{array}{l}\text { Laboratory findings } \\
\text { (reference range) }\end{array}$} \\
\hline & & & & & Bilirubin & ALP & ALT & cPL \\
\hline & & & & & $\begin{array}{l}(2.5- \\
8.5 \mu \mathrm{mol} / \mathrm{l})\end{array}$ & $\begin{array}{l}(33-215 \\
U / I)\end{array}$ & $\begin{array}{l}(18-77 \\
\text { U/I) }\end{array}$ & $\begin{array}{l}(< \\
200 \mu g / l)\end{array}$ \\
\hline $\begin{array}{l}\text { Dog } \\
1 *\end{array}$ & Spanish Water Dog & 8 & 18.9 & Icterus, Vomiting & 786.4 & 1760 & 1574 & - \\
\hline $\begin{array}{l}\text { Dog } \\
2^{\star}\end{array}$ & Red Irish Setter & 9 & 24.8 & Icterus, Vomiting, Diarrhea & 411.2 & 16048 & 1089 & - \\
\hline $\begin{array}{l}\text { Dog } \\
3^{*}\end{array}$ & Giant Poodle & 7 & 18.7 & Icterus, Vomiting, Inappetence & 367.2 & 2318 & 1998 & 496 \\
\hline $\begin{array}{l}\text { Dog } \\
4^{\star}\end{array}$ & $\begin{array}{l}\text { Nova Scotia Duck Tolling } \\
\text { Retriever }\end{array}$ & 6 & 24 & Icterus, Vomiting, Diarrhea & 183.4 & 5833 & 2870 & 103 \\
\hline $\begin{array}{l}\text { Dog } \\
5^{\star}\end{array}$ & Bichon Frise & 6 & 6.7 & Icterus, Vomiting & 176.6 & 6759 & 642 & - \\
\hline $\begin{array}{l}\text { Dog } \\
6^{*}\end{array}$ & Parson Russell Terrier & 11 & 9 & Icterus, Vomiting, Inappetence & 51.6 & 16442 & 2434 & 79 \\
\hline $\begin{array}{l}\text { Dog } \\
7 *\end{array}$ & White Shepherd Dog & 8 & 27.7 & $\begin{array}{l}\text { Icterus, Vomiting, Inappetence, Diarrhea, } \\
\text { Weight loss }\end{array}$ & 49.5 & 5049 & 2369 & 35 \\
\hline $\begin{array}{l}\text { Dog } \\
8^{*}\end{array}$ & $\begin{array}{l}\text { Nova Scotia Duck Tolling } \\
\text { Retriever }\end{array}$ & 6 & 18.5 & Icterus, Vomiting, Inappetence & 45.2 & 1051 & 1837 & 267 \\
\hline $\begin{array}{l}\text { Dog } \\
9^{*}\end{array}$ & French Bulldog & 3 & 6.9 & Icterus, Weight loss & 14 & 11429 & 850 & 217 \\
\hline $\begin{array}{l}\text { Dog } \\
10\end{array}$ & Belgian Shepherd Dog & 2 & 29 & $\begin{array}{l}\text { Vomiting, Abdominal pain, } \\
\text { Weight loss }\end{array}$ & 4.3 & 196 & 23 & - \\
\hline $\begin{array}{l}\text { Dog } \\
11\end{array}$ & Jack Russel Terrier & 3 & 10 & Vomiting, Abdominal pain & 4.3 & 102 & 42 & 1000 \\
\hline $\begin{array}{l}\text { Dog } \\
12\end{array}$ & Shetland Sheepdog & 8 & 5.5 & Vomiting, Abdominal pain & 3.9 & 76 & 77 & - \\
\hline $\begin{array}{l}\text { Dog } \\
13\end{array}$ & Short-haired Collie & 11 & 24.8 & Vomiting, Borborygmus & 3.3 & 173 & 637 & 694 \\
\hline $\begin{array}{l}\text { Dog } \\
14\end{array}$ & Fox Terrier & 11 & 13 & Vomiting, Diarrhea, Abdominal pain & 2.6 & 310 & 32 & - \\
\hline $\begin{array}{l}\text { Dog } \\
15\end{array}$ & Finnish Lapphund & 4 & 20 & Vomiting & 2.5 & 151 & 39 & 118 \\
\hline
\end{tabular}

ALP: Alkaline phosphatase; ALT: Alanine aminotransferase; cPL: canine pancreatic lipase, *: icteric; abnormal results in bold font 
Table 2

Findings of 15 dogs in ultrasound, ERC (including endoscopy) and ERP

\begin{tabular}{|c|c|c|c|c|c|c|c|c|c|c|c|}
\hline \multirow[t]{2}{*}{ Case } & \multirow{2}{*}{$\begin{array}{l}\text { Liver } \\
\text { US }\end{array}$} & \multicolumn{2}{|c|}{ Major papilla } & \multicolumn{2}{|l|}{ Gallbladder } & \multicolumn{2}{|c|}{ Common bile duct } & \multirow{2}{*}{$\begin{array}{l}\begin{array}{l}\text { Extrahepatic } \\
\text { duct }\end{array} \\
\text { ERC }\end{array}$} & \multirow{2}{*}{$\begin{array}{l}\text { Intrahepatic } \\
\text { duct } \\
\text { ERC }\end{array}$} & \multicolumn{2}{|l|}{ Pancreatic area } \\
\hline & & US & $\begin{array}{l}\text { ERC } \\
\text { (Endoscopy) }\end{array}$ & US & ERC & US & ERC & & & $\begin{array}{l}\text { US } \\
\text { (pancreas) }\end{array}$ & $\begin{array}{l}\text { ERP } \\
\text { (ducts }\end{array}$ \\
\hline $\begin{array}{l}\text { Dog } \\
1 *\end{array}$ & $\begin{array}{l}\text { Hypo- } \\
\text { echoic }\end{array}$ & Normal & $\begin{array}{l}\text { Prominent } \\
\text { (open) }\end{array}$ & Normal & Normal & Normal & $\begin{array}{l}\text { Dilated } \\
20 \mathrm{~mm} \text { and } \\
\text { narrowed }\end{array}$ & Normal & Normal & Hyperechoic & NP \\
\hline $\begin{array}{l}\text { Dog } \\
2^{\star}\end{array}$ & $\begin{array}{l}\text { Hyper- } \\
\text { echoic }\end{array}$ & Prominent & Normal & $\begin{array}{l}\text { Distended, } \\
\text { debris }\end{array}$ & $\begin{array}{l}\text { No } \\
\text { filling }\end{array}$ & $\begin{array}{l}\text { Dilated } \\
10 \mathrm{~mm}\end{array}$ & $\begin{array}{l}\text { No filling } \\
\text { (Obstruction) }\end{array}$ & Dilated & Normal & Hyperechoic & Norme \\
\hline $\begin{array}{l}\text { Dog } \\
3^{*}\end{array}$ & $\begin{array}{l}\text { Hyper- } \\
\text { echoic }\end{array}$ & Normal & Normal & $\begin{array}{l}\text { Distended, } \\
\text { debris }\end{array}$ & NR & $\begin{array}{l}\text { Dilated } \\
10 \mathrm{~mm}\end{array}$ & $\begin{array}{l}\text { Dilated } \\
5 \mathrm{~mm} \\
\text { (Obstruction) }\end{array}$ & NR & Dilated & Normal & Norma \\
\hline $\begin{array}{l}\text { Dog } \\
4^{\star}\end{array}$ & $\begin{array}{l}\text { Hyper- } \\
\text { echoic }\end{array}$ & NR & Normal & Normal & Normal & Normal & Normal & Normal & No filling & Normal & Norme \\
\hline $\begin{array}{l}\text { Dog } \\
5^{\star}\end{array}$ & $\begin{array}{l}\text { Hyper- } \\
\text { echoic }\end{array}$ & $N R$ & Normal & $\begin{array}{l}\text { Distended, } \\
\text { debris }\end{array}$ & Normal & Normal & Narrowed & $\begin{array}{l}\text { Dilated } \\
5 \mathrm{~mm}\end{array}$ & Normal & Hypoechoic & NP \\
\hline $\begin{array}{l}\text { Dog } \\
6^{*}\end{array}$ & $\begin{array}{l}\text { Hetero- } \\
\text { genic }\end{array}$ & Prominent & Prominent & $\begin{array}{l}\text { Distended, } \\
\text { debris }\end{array}$ & NR & $\begin{array}{l}\text { Dilated } \\
4.5 \mathrm{~mm}\end{array}$ & Dilated & Dilated & NR & Heterogenous & NP \\
\hline $\begin{array}{l}\text { Dog } \\
7^{\star}\end{array}$ & $\begin{array}{l}\text { Hyper- } \\
\text { echoic }\end{array}$ & NR & Normal & $\begin{array}{l}\text { Distended, } \\
\text { debris }\end{array}$ & $\begin{array}{l}\text { No } \\
\text { filling }\end{array}$ & $\begin{array}{l}\text { Dilated } \\
5.3 \mathrm{~mm}\end{array}$ & No filling & $\begin{array}{l}\text { Dilated } \\
10 \mathrm{~mm}\end{array}$ & NR & Hyperechoic & NP \\
\hline $\begin{array}{l}\text { Dog } \\
8^{*}\end{array}$ & $\begin{array}{l}\text { Hyper- } \\
\text { echoic }\end{array}$ & Prominent & Prominent & $\begin{array}{l}\text { Distended, } \\
\text { debris }\end{array}$ & NR & $\begin{array}{l}\text { Dilated } \\
9 \mathrm{~mm}\end{array}$ & $\begin{array}{l}\text { Dilated } \\
10 \mathrm{~mm}\end{array}$ & NR & NR & Normal & NP \\
\hline $\begin{array}{l}\text { Dog } \\
9 *\end{array}$ & $\begin{array}{l}\text { Hypo- } \\
\text { echoic }\end{array}$ & NR & Normal & Normal & Normal & Normal & Normal & Normal & Normal & Hyperechoic & Norma \\
\hline $\begin{array}{l}\text { Dog } \\
10\end{array}$ & Normal & NR & Normal & Normal & Normal & Normal & $\begin{array}{l}\text { Abnormal } \\
\text { course }\end{array}$ & NR & NR & Normal & NP \\
\hline $\begin{array}{l}\text { Dog } \\
11\end{array}$ & Normal & NR & Stenosis & Normal & Normal & Normal & Normal & Normal & NR & Hypoechoic & Norme \\
\hline $\begin{array}{l}\text { Dog } \\
12\end{array}$ & Normal & NR & Very small & $\begin{array}{l}\text { Distended, } \\
\text { mucocele }\end{array}$ & NR & Normal & Normal & Normal & Normal & Hyperechoic & NP \\
\hline $\begin{array}{l}\text { Dog } \\
13\end{array}$ & Normal & NR & Stenosis & $\begin{array}{l}\text { Distended, } \\
\text { debris }\end{array}$ & Normal & $\begin{array}{l}\text { Dilated } \\
10 \mathrm{~mm}\end{array}$ & $\begin{array}{l}\text { Dilated } \\
10 \mathrm{~mm}\end{array}$ & NR & NR & Normal & NP \\
\hline $\begin{array}{l}\text { Dog } \\
14\end{array}$ & Normal & Prominent & Normal & $\begin{array}{l}\text { Distended, } \\
\text { debris }\end{array}$ & Normal & Normal & Normal & Normal & $\begin{array}{l}\text { Decreased } \\
\text { filling }\end{array}$ & Hyperechoic & Norma \\
\hline $\begin{array}{l}\text { Dog } \\
15\end{array}$ & Normal & NR & Normal & Normal & Normal & Normal & Normal & NR & NR & Hypoechoic & $\begin{array}{l}\text { Abnorı } \\
\text { course }\end{array}$ \\
\hline
\end{tabular}


Table 3

Histopathologic findings of the liver, pancreas, and duodenum of 15 dogs that underwent ERC/ERCP and/or EST

\begin{tabular}{|c|c|c|c|c|}
\hline Dog & $\begin{array}{l}\text { Type of } \\
\text { sampling }\end{array}$ & Hepatobiliary system & Pancreas & Duodenum \\
\hline $\begin{array}{l}\text { Dog } \\
1 *\end{array}$ & $\begin{array}{l}\text { Biopsy } \\
\text { Laparoscopy }\end{array}$ & $\begin{array}{l}\text { Moderate chronic neutrophilic cholangiohepatitis, Biliary } \\
\text { stasis }\end{array}$ & $\begin{array}{l}\text { Focal severe fibrosig } \\
\text { pancreatitis with multifocal } \\
\text { moderate peripancreatic } \\
\text { chronic histiocytic steatitis }\end{array}$ & Not sampled \\
\hline $\begin{array}{l}\text { Dog } \\
2^{*}\end{array}$ & Autopsy & $\begin{array}{l}\text { Moderate chronic neutrophilic cholangiohepatits, } \\
\text { Moderate biliary stasis, Pancreatic carcinoma metastasis } \\
\text { in the liver }\end{array}$ & Exocrine pancreatic carcinoma & $\begin{array}{l}\text { Infiltration of pancreatic } \\
\text { carcinoma into the duodenal wall } \\
\text { compressing the common bile } \\
\text { duct }\end{array}$ \\
\hline $\begin{array}{l}\text { Dog } \\
3^{\star}\end{array}$ & $\begin{array}{l}\text { Biopsy } \\
\text { Endoscopy }\end{array}$ & Not sampled & Not sampled & $\begin{array}{l}\text { Moderate chronic diffuse } \\
\text { lymphoplasmacytic enteritis }\end{array}$ \\
\hline $\begin{array}{l}\text { Dog } \\
4^{*}\end{array}$ & $\begin{array}{l}\text { Biopsy } \\
\text { Laparoscopy }\end{array}$ & $\begin{array}{l}\text { Severe chronic neutrophilic and fibrosing } \\
\text { cholangiohepatitis }\end{array}$ & Not sampled & Not sampled \\
\hline $\begin{array}{l}\text { Dog } \\
5^{\star}\end{array}$ & $\begin{array}{l}\text { Biopsy } \\
\text { Laparotomy }\end{array}$ & $\begin{array}{l}\text { Severe diffuse chronic hepatic lipidosis and multifocal } \\
\text { extramedullary hematopoiesis }\end{array}$ & $\begin{array}{l}\text { No significant parenchymal } \\
\text { changes, Mild interstitial } \\
\text { fibrosis, Acute endothelial } \\
\text { swelling }\end{array}$ & Not sampled \\
\hline $\begin{array}{l}\text { Dog } \\
6^{*}\end{array}$ & $\begin{array}{l}\text { Biopsy } \\
\text { Laparoscopy }\end{array}$ & $\begin{array}{l}\text { Moderate multifocal chronic neutrophilic and fibrosing } \\
\text { hepatitis, Moderate chronic lymphoplasmacytic } \\
\text { cholecystitis with mild cystic mucinous hyperplasia of } \\
\text { gallbladder mucosa }\end{array}$ & Not sampled & Not sampled \\
\hline $\begin{array}{l}\text { Dog } \\
7^{*}\end{array}$ & Autopsy & $\begin{array}{l}\text { Moderate chronic neutrophilic and fibrosing } \\
\text { cholangiohepatitis, Moderate cystic mucinous } \\
\text { hyperplasia of gallbladder mucosa }\end{array}$ & $\begin{array}{l}\text { No significant histological } \\
\text { changes }\end{array}$ & $\begin{array}{l}\text { No significant histological } \\
\text { changes }\end{array}$ \\
\hline $\begin{array}{l}\text { Dog } \\
8^{*}\end{array}$ & Autopsy & $\begin{array}{l}\text { Moderate chronic fibrosing and neutrophilic } \\
\text { cholangiohepatits }\end{array}$ & $\begin{array}{l}\text { No significant histological } \\
\text { changes }\end{array}$ & $\begin{array}{l}\text { Local severe chronic } \\
\text { lymphoplasmacytic mural } \\
\text { enteritis at papilla }\end{array}$ \\
\hline $\begin{array}{l}\text { Dog } \\
9 *\end{array}$ & $\begin{array}{l}\text { Biopsy } \\
\text { Laparoscopy }\end{array}$ & $\begin{array}{l}\text { Moderate chronic fibrosing and neutrophilic } \\
\text { cholangiohepatits (Autopsy: Focal abscess and severe } \\
\text { biliary stasis) }\end{array}$ & Not sampled & $\begin{array}{l}\text { No significant histological } \\
\text { changes }\end{array}$ \\
\hline $\begin{array}{l}\text { Dog } \\
10\end{array}$ & Autopsy & $\begin{array}{l}\text { Moderate multifocal fibrosing and histiocytic } \\
\text { inflammation involving areas around duodenum, } \\
\text { pancreas and a curved common bile duct, Hepatic biliary } \\
\text { stasis }\end{array}$ & $\begin{array}{l}\text { Severe peripancreatic chronic } \\
\text { histiocytic steatitis }\end{array}$ & $\begin{array}{l}\text { No significant histological } \\
\text { changes }\end{array}$ \\
\hline $\begin{array}{l}\text { Dog } \\
11\end{array}$ & $\begin{array}{l}\text { Biopsy } \\
\text { Endoscopy }\end{array}$ & Not sampled & Not sampled & $\begin{array}{l}\text { Mild chronic diffuse eosinophilic } \\
\text { enteritis }\end{array}$ \\
\hline $\begin{array}{l}\text { Dog } \\
12\end{array}$ & & Not sampled & Not sampled & Not sampled \\
\hline $\begin{array}{l}\text { Dog } \\
13\end{array}$ & $\begin{array}{l}\text { Biopsy } \\
\text { Endoscopy }\end{array}$ & Not sampled & Not sampled & $\begin{array}{l}\text { Mild chronic diffuse } \\
\text { lymphoplasmacytic enteritis with } \\
\text { local moderate acute } \\
\text { fibrinonecrotizing duodenitis }\end{array}$ \\
\hline $\begin{array}{l}\text { Dog } \\
14\end{array}$ & Autopsy & Mild hepatic fibrosis, Mild acute necrotizing cholecystitis & $\begin{array}{l}\text { Acute severe multifocal } \\
\text { necrotizing pancreatitis }\end{array}$ & $\begin{array}{l}\text { Local severe necrotizing } \\
\text { peritonitis, Moderate diffuse } \\
\text { chronic lymphoplasmacytic } \\
\text { enteritis }\end{array}$ \\
\hline $\begin{array}{l}\text { Dog } \\
15\end{array}$ & $\begin{array}{l}\text { Biopsy } \\
\text { Endoscopy }\end{array}$ & Not sampled & Not sampled & $\begin{array}{l}\text { Mild diffuse chronic } \\
\text { lymphoplasmacytic enteritis }\end{array}$ \\
\hline
\end{tabular}

\section{Discussion}

All 15 dogs of this study revealed some abnormal findings in US and ERC/ERCP examinations. The most agreement between US and ERC occurred for assessing the normality or abnormality of the CBD. In case of disagreement of both modalities, the ERC findings of the ductal structures were in line with the available laparotomy, laparoscopy, or autopsy findings more often than the US findings. In contrary, for the gallbladder the US findings were closer to the laparotomy, laparoscopy, or autopsy findings. In a human study, US was in agreement with abnormal ERCP findings in 101 of 120 patients (sensitivity $84 \%$ ) and of normal ERCP findings in 70 of 73 patients (specificity 95\%) [16]. US has been shown to be more sensitive in diagnosis of dilated or strictured bile ducts than finding choledocholithiasis [16]. ERCP is considered complimentary to US specifically when the problem is located within the pancreatobiliary ductal anatomy.

The comparison of US and ERC results revealed different degrees in agreement and disagreement dependent on the reported anatomical structure. The major papilla was visible in all 15 dogs scanned by endoscopy for ERC examination, but was reported only in six dogs by using US. This is in agreement with a previous study, which found that the major papilla was visualized ultrasonographically in $42 \%$ of dogs without evidence of abdominal disorders [17]. The 
visibility was negatively influenced by the presence of air or food in the gastrointestinal tract and increased body weight [17]. US and endoscopy for ERC/ERCP disagreed concerning the major papilla in three dogs of our study, which may be due to contractile activity of the sphincter of Oddi [18] or different types of the major papilla [19]. Since no histopathology reports were available for the major papilla in these three dogs, however, it is impossible to show which modality had the correct diagnosis.

US and ERC agreed in a normal or dilated CBD in 10/15 dogs. A recent study showed that US is a useful modality for detection of the canine CBD diameter and that US results were not significantly different from those of CT [20]. In human medicine, bile duct dilatation has been detected by US with the sensitivity of $85-95 \%$ for experienced examiners [21]. In all three dogs (Dogs 1, 5, 10) that showed normal CBD in US but abnormal in ERC, pathology revealed abnormal findings of the hepatobiliary system which highlights the fact that a normal US examination cannot rule out the need for ERC. Two dogs (Dogs 2 and 7) had a dilated CBD in US but the duct did not fill with contrast material in ERC. In Dog 2, this was found to be due to infiltration of carcinoma within the intestinal wall, causing pressure to the duct, and in the other dog, precipitated bile was detected within the gallbladder at autopsy. MRCP could be helpful in cases where space-occupying masses prevent contrast material visibility within the biliary ducts [4].

The gallbladder was visible and could be assessed more often by US than by ERC, and the US findings were more in agreement with the laparotomy, laparoscopy, or autopsy findings where available. It can be speculated that the diagnostic value of US is preferentially for the gallbladder, and ERC for ductal structures. In human medicine, it has been shown that US has high sensitivity for detecting cholecystitis [22]. Another study showed that CT was significantly more sensitive for diagnosing cholecystitis than US [23], but because of the speed and portability, US is still used as the initial imaging technique for evaluating patients with suspected acute cholecystitis.

Extrahepatic ducts were not mentioned in any of the US reports. Extrahepatic ducts are usually poorly visualized owing to overlying bowel gas and the normal intrahepatic ducts are not visible in US [24]. Dilated intrahepatic ducts were reported in one dog in US, but ERCP revealed an actual dilation of extrahepatic ducts. Since ERCP has the ability to show the entire biliary system in one view, it can be more reliable in localizing the abnormality.

The technical success rate of ERC and ERP was $88.2 \%$ and $66.7 \%$, respectively. This is comparable with previously reported success rates of $67-75 \%$ for ERC in dogs and cats and of $70 \%$ for ERP in dogs $c 8,9,10$ ]. Failures have been reported to be related to difficulties in the duodenal papilla cannulation in a smallsized dog, intraduodenal contents, changes of the duodenal mucosa, and decrease in gastric tone [9].

In the six dogs that underwent ERP, the duodenal and gastric branches of the main pancreatic ducts were clearly visible with no signs of duct abnormalities in five dogs. In one dog (Dog 15), with serum cPL concentration in the reference range, the duodenal branch of the pancreatic duct did not go caudally but cranially to the diaphragm. In humans, abnormal contrast pancreatograms are used to grade the stage of chronic pancreatitis by the Cambridge classification system from mild to severe [25]. It remained open whether the ERP finding of the dog in our study was a variation of normality, an artifact, or a possible pathologic finding, since the dog underwent no direct examination of the pancreas by invasive diagnostic methods.

The success rate of EST was $81.8 \%$ with no major procedure-related complications during or immediately after the procedure. In humans, biliary EST is associated with several complications, both in the short- and the long-term [13,14]. Due to the invasive nature of the procedure, these complications are inevitable and observed in some patients, depending on patient- and procedure-related factors [11]. The short-term complications of EST are bleeding, perforation, pancreatitis and cholangitis $[13,14]$. Early identification and appropriate management of complications is essential to reduce mortality and morbidity. Of the known short-term complications of EST, only mild bleeding occurred in one dog of our study. In human medicine, acute pancreatitis is the most common post-ERCP complication, with an incidence of 2-10\% [26]. One dog (Dog 14) showed acute severe necrotizing pancreatitis six months after EST, but there are no laboratory or clinical records of the dogs until six months after EST. It can be suggested that an acute pancreatitis developing six months after EST is unlikely to have been caused by the procedure.

Only four of nine dogs showed clinical and laboratory improvement after EST. This finding is similar to the findings of previous studies reporting clinical improvement after EST in a dog with papillary stenosis [9] and in one dog with signs of extrahepatic duct obstruction treated by EST and biliary stenting [10]. One dog (Dog 6), regardless of clinical and laboratory improvement after EST, developed recurrent fever four months after EST and bacterial culture revealed ESBL E. coli in the bile and feces. A study in humans showed that the prevalence of post-ERCP biliary tract infections was $4 \%$ of the patients [27]. It remained open whether the ESBL E.coli infection in Dog 6 was a complication of ERC or an ascending infection from intestine after EST.

The finding of dilated CBD in ERC without evidence of obstruction in two icteric dogs, was of interest. The cause of this dilation could not be identified. However, after EST there was marked bile flow into the duodenum. A report on six cats with sphincter of Oddi pathology speculated that patients without obvious pathology at the papilla might have sphincter dysfunction with extrahepatic biliary obstructions due to a chronic inflammatory enteropathy [28]. Histopathology of the duodenal biopsy samples in Dog 8 revealed mural enteritis at the papilla.

Four dogs underwent EST without improvement and were euthanized within days after the procedure. Clear causes of posthepatic and intrahepatic cholestasis were established by autopsy in three dogs. Dog 2 had an exocrine pancreatic carcinoma infiltrating the duodenal wall, Dog 7 a moderate chronic cholangiohepatitis and cystic mucinous hyperplasia of the gallbladder mucosa and Dog 8 moderate chronic cholangiohepatitis and severe chronic mural enteritis. No clear cause was found for Dog 5 with a CBD stricture and increasing hyperbilirubinemia after EST, since hepatic lipidosis was the sole histopathological finding in the liver. Prolonged cholestasis after ERC may be one possible reason. It is a very rare but recognized complication following ERCP in humans [29]. The exact mechanism for prolonged jaundice post-ERCP has remained unclear but it has been suggested that it may be directly related to the radiocontrast medium, perhaps due to an idiosyncratic adverse reaction resulting in disruption of the hepatocyte canalicular membrane or interruption of transport pumps with subsequent intrahepatic cholestasis and jaundice [29, 30]. 
Findings of patients without short-term improvement after EST, and the occurrence of an infection with ESBL, acute necrotizing pancreatitis and biliary peritonitis within 3 to 10 months after EST revealed that EST can be of no help or has the possibility to harm the patient in the short or long run. The indication for EST should be the result of a thorough diagnostic work up of the individual patient suspect for a biliary tract disorder to rule out hepatic or pancreatic diseases requiring different therapeutic approaches. The study revealed that ERC has some advantages over US in visualizing pathologies of the biliary ductal system, but it is technically demanding and with some risk of complications. MRCP is a less invasive alternative to diagnostic ERC since it was shown to have good accuracy for the diagnosis of hepatobiliary and pancreatic duct disorders in dogs [4]. MRCP could be a beneficial imaging tool for selecting patients that require ERC and subsequent EST. MRCP does not only help to visualize the biliary and pancreatic duct systems but also the surrounding soft tissues which is impossible with ERCP. As established in human medicine, endoscopic treatment should include biliary stenting when diagnosing distal or long CBD strictures by MRCP or ERC, especially with malignant lesions that are not eligible for open surgery [31]. Endoscopic CBD stenting has been proven possible in dogs [10]. It is very likely that the establishment of MRCP in the diagnostic work up of dogs with suspected disorders of the biliary duct system could give a better indication and a more successful outcome of endoscopic interventions [4].

There are some limitations in the present study. The retrospective nature of the study caused lack of systematic reports for US and ERC/ERCP and EST. The US reports were written for clinical purposes and by different veterinary radiologists. Lack of systematic collection of laboratory, ultrasonographic and clinical follow-up data after the procedures made comparison difficult in some cases. There was also a lack of histopathologic confirmation of the lesions in some patients.

\section{Conclusion}

Findings from this study indicate that US is an important initial imaging test in the evaluation of dogs presenting with gastrointestinal signs suggestive for a disease of the hepatobiliary system. US allows very good assessment of the gallbladder but has some limitations in assessing the papillae as well as the extra- and intrahepatic biliary duct system. ERC can serve as a complementary diagnostic procedure to improve the assessment of the biliary duct system but has limitations to assess the gallbladder and is technically demanding with risks of serious complications. EST is technically possible and offers a minimally invasive technique to treat obstructive biliary diseases in dogs. It can, however, be non-successful if the indication is not set right for the individual patient or cause serious short- and long-term complications. This demands a structured diagnostic and risk-benefit-assessment approach that is currently missing. There is also a need for more advanced diagnostic imaging of the biliary duct system, such as MRCP, to improve the selection for ERC and EST.

\section{Methods}

This study is a retrospective descriptive case series. Electronic medical records of canine patients examined between November 2007 and March 2019 were searched from data archives of the Small Animal Hospital, Faculty of Veterinary Medicine, University of Helsinki. The search for patients included the terms "ERCP", "ERC" and "Fluoroscopy". The criteria for inclusion of dogs were a successfully performed diagnostic ERC with or without ERP, or therapeutic EST.

The clinical records, laboratory test results (serum biochemical profile: bilirubin, ALP, ALT, CPL), US findings, ERC/ERCP findings and histopathological data of the patients were reviewed. In addition, short-term and, if available, long-term outcomes after the therapeutic EST procedure were obtained from the medical records. All dogs were treated medically based on their clinical problems, but these treatments are beyond the scope of this article.

\section{Abdominal Ultrasonography}

Abdominal US had been performed in all patients prior to ERC/ERCP, using an iU22 Ultrasound System (Philips, Bothell, WA) for all cases except for one dog, which had been examined using an EPIQ 7 Ultrasound System (Philips, Bothell, WA). The reports written by the examining radiologist formed the material for the present study. The following aspects were included: The liver echogenicity, compared to the falciform fat, was classified as normal, hyperechoic, hypoechoic or heterogenous. The biliary system had been evaluated for the presence of prominent major papilla, presence of distended gallbladder with debris, CBD (including diameter measurement, when available), and dilated extra- and intrahepatic ducts. The CBD was considered normal when the diameter was $\leq 3 \mathrm{~mm}[24,32]$. Echogenicity of the pancreas had been compared to peripancreatic mesenteric fat and was classified as normal, hyperechoic,

\section{hypoechoic or heterogenous.}

\section{Endoscopic procedures}

Endoscopic procedures had been performed under general anesthesia based on previously published protocols in dogs [5, 9]. Based on these protocols, conventional gastroduodenoscopy was performed by using a standard flexible videoscope on dogs in left recumbency. ERCP was performed in ventral recumbency with an 11.0-mm side-view endoscope (JF1T10, Olympus, Tokyo, Japan) in dogs > $10 \mathrm{~kg}$ body weight and an 7.5-mm side-view endoscope (PJF 160 , Olympus, Tokyo, Japan) in dogs $<10 \mathrm{~kg}$ body weight. The major and minor papillae were cannulated by inserting a sphincterotome catheter into the papillae. The sphincterotome Clever Cut 3V (KD-V431M-0720, Olympus, Tokyo, Japan) was used for dogs > 10 kg body weight and the sphincterotome REF OE1051615GW (Endoflex GmbH, Voerde, Germany) for dogs $<10 \mathrm{~kg}$ body weight. The following aspects were included in the endoscopic and ERC/ERCP evaluation: prominent or stenotic major or minor papilla; abnormal, narrowed or dilated CBD based on reported normal range at different sites of the duct [5]; abnormal gallbladder filling; dilated extrahepatic ducts; dilated or abnormal filling of the intrahepatic ducts; and abnormal pancreatic duct. For contrast studies of both ductal systems, the sphincterotome catheter was filled with iodine contrast medium (lomeprol 300, Bracco-Byk Gulden, Konstanz, Germany) before the procedure. In cases of papillary stenosis or blocked bile flow from the major papilla, EST was performed by the respective sphincterotome supported by a guidewire. For dogs $>10 \mathrm{~kg}$ the guidewire VisiGlide G-240-2545S (Olympus, Tokyo, Japan) was used and for dogs $<10 \mathrm{~kg}$ the guidewire StripeGuide REF 21525400 (Endoflex GmbH, Voerde, Germany). For EST of the major papilla, the tip of the sphincterotome with its diathermy wire was inserted into the papilla and bowed to bring the diathermy wire into cutting position. Then the papilla was cut open electrosurgically in steps using a electrosurgical generator (ESG-100, Olympus, Tokyo, Japan) until the bowed sphincterotome slid easily through the orifice. 
In case the procedures had been performed more than once on the same animal, only the first session of each procedure was included when reporting the success rate of the procedures. However, all repeated procedures and their outcomes are also reported here.

\section{Histopathological examination}

Samples of liver, pancreas and/or duodenum, obtained either as endo- or laparoscopic biopsies, or at laparotomy, or autopsy, were available as hematoxylineosin stained histological sections of formalin-fixed, paraffin-embedded tissue in 14 of 15 dogs. These samples were re-examined for the present study.

\section{Abbreviations}

US: ultrasonography, ERCP: endoscopic retrograde cholangiopancreatography, ERC: endoscopic retrograde cholangiography, ERP: endoscopic retrograde pancreatography, EST: endoscopic sphincterotomy, MRCP: magnetic resonance cholangiopancreatography, ALP: alkaline phosphatase, ALT: alanine aminotransferase, CPL: canine pancreatic lipase, CBD: common bile duct, ESBL: extended spectrum beta-lactamase

\section{Declarations}

Ethics approval and consent to participate: All diagnostic and treatment procedures in our study were part of the regular clinical work-up of the patients at Teaching Small Animal Hospital of the University of Helsinki. In accordance to the Finnish national regulations

(https://finlex.fi/en/laki/kaannokset/2013/20130497) the non-experimental clinical veterinary practice is excluded from the scope of legislation and therefore ethical approval was not required for this study.

Consent for publication: Not applicable

Availability of data and materials: The datasets supporting the conclusions of this article are included within the article.

Competing interests: The authors declare that they have no competing interests

Funding: Doctoral program of the clinical veterinary medicine (DP-CVM) of the Helsinki University supported the personal grant of the first author (VR) and has had no other role in this research project or any other related procedures.

\section{Authors' contributions:}

VR: Gathering data, Drafting the manuscript, Revision; TS: ERCPs, Drafting the manuscript, Revision; JH: ERCPs, Revision; PS: Pathology, Revision; MR: Drafting the manuscript, Revision. All authors read, reviewed, and approved the final manuscript.

Acknowledgements: None

\section{References}

1. Center SA. Diseases of the Gallbladder and Biliary Tree. Vet Clin North Am - Small Anim Pract. 2009;39(3):543-98.

2. Lidbury JA, Suchodolski JS. New advances in the diagnosis of canine and feline liver and pancreatic disease. Vet J. 2016;215:87-95.

3. Pratschke KM, Ryan J, Mcalinden A, Mclauchlan G. Pancreatic surgical biopsy in 24 dogs and 19 cats: Postoperative complications and clinical relevance of histological findings. J Small Anim Pract. 2015;56(1):60-6.

4. Marolf AJ. Diagnostic Imaging of the Hepatobiliary System: An Update. Vet Clin North Am - Small Anim Pract. 2017;47(3):555-68.

5. Spillmann T, Happonen I, Kähkönen T, Fyhr T, Westermarck E. Endoscopic retrograde cholangio-pancreatography in healthy Beagles. Vet Radiol Ultrasound. 2005;46(2):97-104.

6. Hong J, Pan W, Zuo W, Wang A, Zhu L, Zhou X, et al. Efficacy and safety of therapeutic ERCP in patients with ectopic papilla of Vater. Medicine. 2020. https://doi.org/ 10.1097/MD.0000000000018536.

7. Spillmann T, Happonen I, Sankari S, Wittker A, Kähkönen T, Westermarck E. Evaluation of serum values of pancreatic enzymes after endoscopic retrograde pancreatography in dogs. Am J Vet Res. 2004;65(5):616-9.

8. Spillmann T, Willard MD, Ruhnke I, Suchodolski JS, Steiner JM. Feasibility of endoscopic retrograde cholangiopancreatography in healthy cats. Vet Radiol Ultrasound. 2014;55(1):85-91.

9. Spillmann T, Schnell-Kretschmer H, Dick M, Gröndahl KA, Lenhard TCW, Rüst SK. Endoscopic retrograde cholangio-pancreatography in dogs with chronic gastrointestinal problems. Vet Radiol Ultrasound. 2005;46(4):293-9.

10. Berent A, Weisse C, Schattner M, Gerdes H, Chapman P, Kochman M. Initial experience with endoscopic retrograde cholangiography and endoscopic retrograde biliary stenting for treatment of extrahepatic bile duct obstruction in dogs. J Am Vet Med Assoc. 2015;246(4):436-46.

11. Köksal A, Eminler AT, Parlak E. Biliary endoscopic sphincterotomy: Techniques and complications. World J Clin Cases. 2018;6(16):1073-86.

12. Cotton PB, Lehman G, Vennes J, Geenen JE, Russell RCG, Meyers WC, et al. Endoscopic sphincterotomy complications and their management: an attempt at consensus. Gastrointest Endosc. 1991;37(3):383-93.

13. Cotton PB, Garrow DA, Gallagher J, Romagnuolo J. Risk factors for complications after ERCP: a multivariate analysis of 11,497 procedures over 12 years. Gastrointest Endosc. 2009;70(1):80-8.

14. Thomas R, Wojda JD, Nuschke, Ellyn A, Smith R, Modi S, Schadt D, Strosberg. Berhanu Geme, Sagar C Galwankar SPS. Endoscopic retrograde cholangiography: Complications, emergencies, and related topics. Int J Acad Mrdicine. 2018;4(2):124-41.

Page $10 / 13$ 
15. Moffatt DC, Yu BN, Yie W, Bernstein CN. Trends in utilization of diagnostic and therapeutic ERCP and cholecystectomy over the past 25 years: A population-based study. Gastrointest Endosc. 2014;79(4):615-22.

16. Lindsell DRM. Ultrasound imaging of pancreas and biliary tract. Lancet. 1990;335:390-3.

17. Barberet V, Schreurs E, Rademacher N, Nitzl D, Taeymans O, Duchateau L, et al. Quantification of the effect of various patient and image factors on ultrasonographic detection of select canine abdominal organs. Vet Radiol Ultrasound. 2008;49(3):273-6.

18. Scott RB, Strasberg SM, El-Sharkawy TY, Diamant NE. Fasting canine biliary secretion and the sphincter of Oddi. Gastroenterology. 1984;87(4):793-804.

19. Adler DG. ERCP biliary cannulation difficulty as a function of papillary subtypes: a tale of shapes and Shar-Pei dogs. Gastrointest Endosc. 2019;90(6):964-5.

20. Park HY, Cho YG, Lee YW, Choi HJ. Evaluation of gallbladder and common bile duct size and appearance by computed tomography in dogs. J Vet Sci. 2018;19(5):653-9.

21. Yarmenitis SD. Ultrasound of the gallbladder and the biliary tree. Eur Radiol. 2002;12(2):270-82.

22. Pinto A, Reginelli A, Cagini L, Coppolino F, lanora AAS, Bracale R, et al. Accuracy of ultrasonography in the diagnosis of acute calculous cholecystitis: Review of the literature. Crit Ultrasound J. 2013;5(SUPPL.1):1-4.

23. Joss R, Wertz JM, Lopez. David Olson W. T. Comparing the Diagnostic Accuracy of Ultrasound and CT in Evaluating Acute Cholecystitis. AJR Am J Roentgenol. 2018;211(2):92-7.

24. d'Anjou MA, Penninck DG. Liver. In: Penninck DG, d'Anjou MA: Atlas of small animal ultrasonography, 2nd ed. Aimes, IA: Blackwell, $2005,183-238$.

25. Cotton PB, Williams CB. Endoscopic retrograde cholangio-pancreatography. In: Cotton PB, Williams CB, editors: Practical gastrointestinal endoscopy, 4th ed. Oxford: Blackwell Science, 1996;105 - 86.

26. Thaker AM, Mosko JD, Berzin TM. Post-endoscopic retrograde cholangiopancreatography pancreatitis. Gastroenterol Rep. 2015;3(1):32-40.

27. Du M, Suo J, Liu B, Xing Y, Chen L, Liu Y. Post-ERCP infection and its epidemiological and clinical characteristics in a large Chinese tertiary hospital: A 4year surveillance study. Antimicrob Resist Infect Control. 2017;6(1):1-6.

28. Furneaux RW. A series of six cases of sphincter of Oddi pathology in the cat (2008-2009). J Feline Med Surg. 2010;12(10):794-801.

29. Patani O, Foulkes SL, Njie R, Aspinall RJ. Prolonged cholestasis induced by endoscopic retrograde cholangiopancreatography. Frontline Gastroenterol. 2010;1(2):121-4.

30. Hannah M, Lee PA, Bonis MMK. Persistent cholestatic jaundice after ERCP. Am J Gastroenterol. 2006;101:204-5.

31. Okano N, Igarashi Y, Kishimoto Y, Mimura T, Ito K. Necessity for endoscopic sphincterotomy for biliary stenting in cases of malignant biliary obstruction. Dig Endosc. 2013;25(SUPPL.2):122-5.

32. Zeman KR, Taylor WJK, Rosenfield TA, Schwartz A, Gold AJ. Acute experimental biliary obstruction in the dog: sonographic findings and clinical indications. Am J Roentgenol. 1981;136:965-7.

\section{Figures}
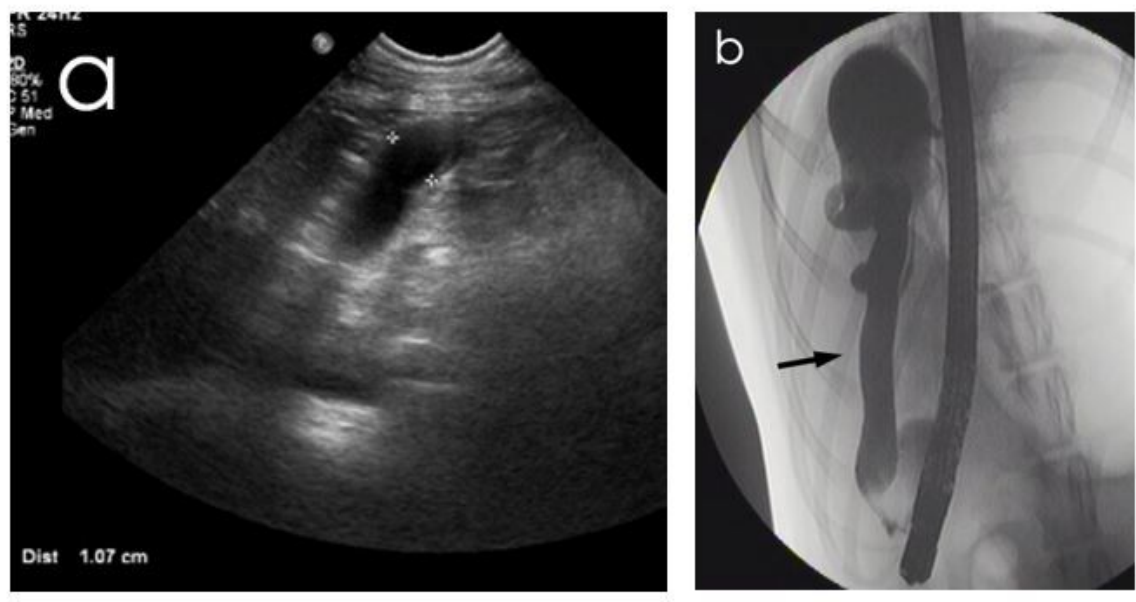

Figure 1

Images of a non-icteric 11-year-old male Short-haired Collie (Dog 13). a) Ultrasonographic image acquired before ERC, showing dilatation of the common bile duct (cursors). b) Fluoroscopic image acquired during ERC, representing dilatation of the common bile duct (arrow, maximum diameter 10 mm). 

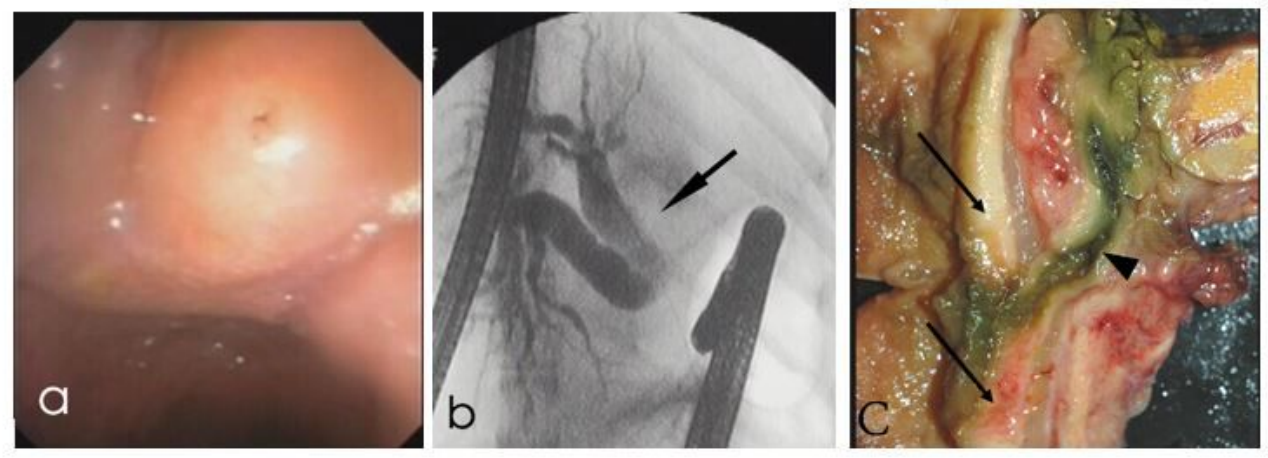

\section{Figure 2}

Images of an icteric 9-year-old female Red Irish Setter (Dog 2). a) Endoscopic image of the prominent major papilla. b) Fluoroscopic image acquired during ERC showing marked dilatation of the extrahepatic ducts (arrow) without contrast filling of the common bile duct. c) Thickened duodenal wall due to Infiltrative carcinoma (arrows) around the common bile duct (arrowhead) at autopsy one day after ERC and EST.
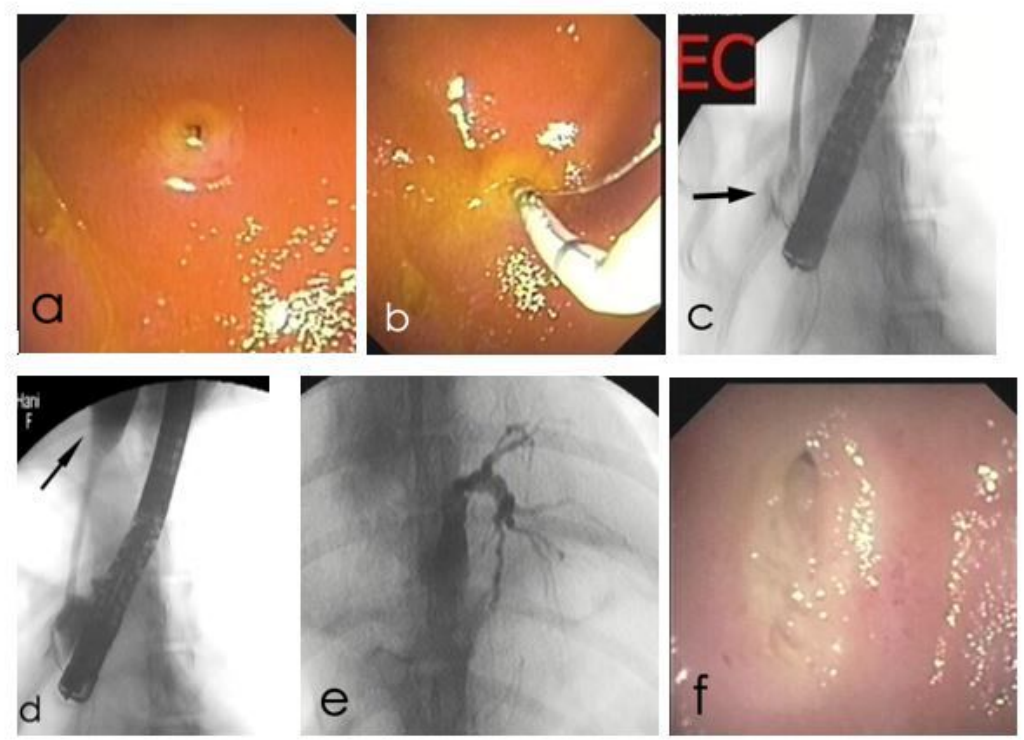

\section{Figure 3}

Images of an icteric 7-year-old female Giant Poodle (Dog 3). a) Endoscopic image of the major papilla before EST. b) Endoscopic image while the sphincterotomy catheter is entering the major papilla. c) Fluoroscopic image acquired during ERC, showing radiolucent concretions (arrow) at the beginning of the common bile duct. d) Fluoroscopic image showing dilatation of the common bile duct (arrow, maximum diameter $5 \mathrm{~mm}$ ). e) Fluoroscopic image representing marked dilatation of the intrahepatic duct. f) Endoscopic image of the major papilla three weeks after EST. 


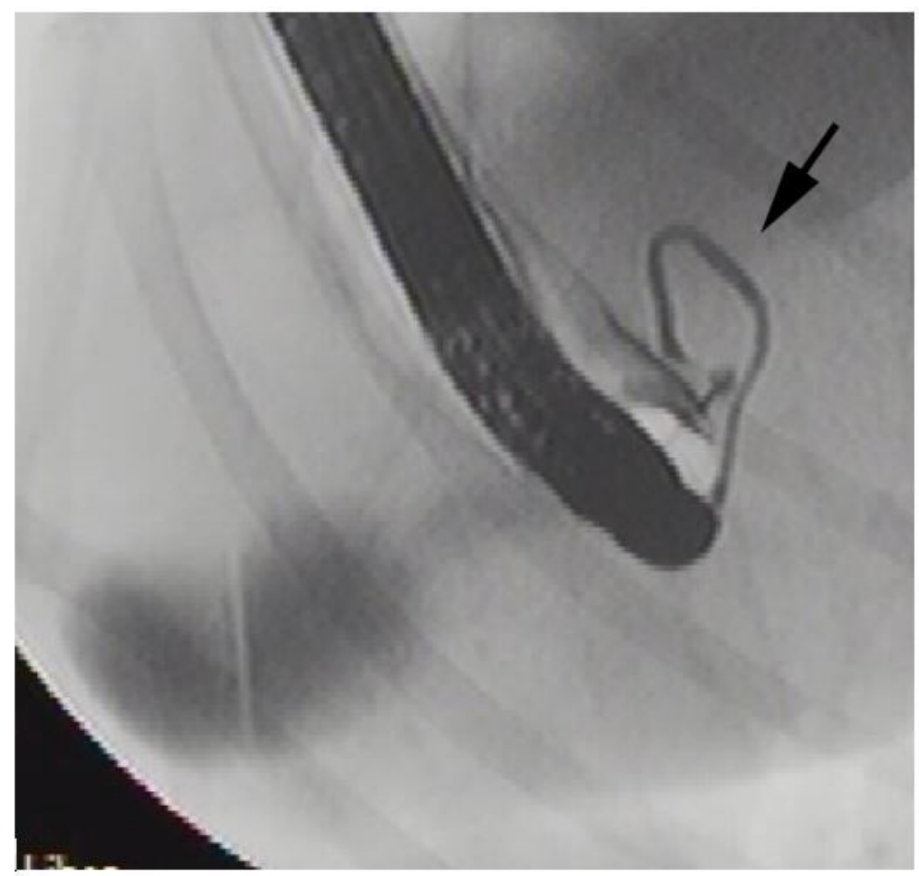

\section{Figure 4}

Fluoroscopic image of a non-icteric 2-year-old male Belgian shepherd dog (Dog 10) acquired during ERC representing abnormal course of the CBD (arrow) in a half circle around the duodenum and not straight to the gallbladder.

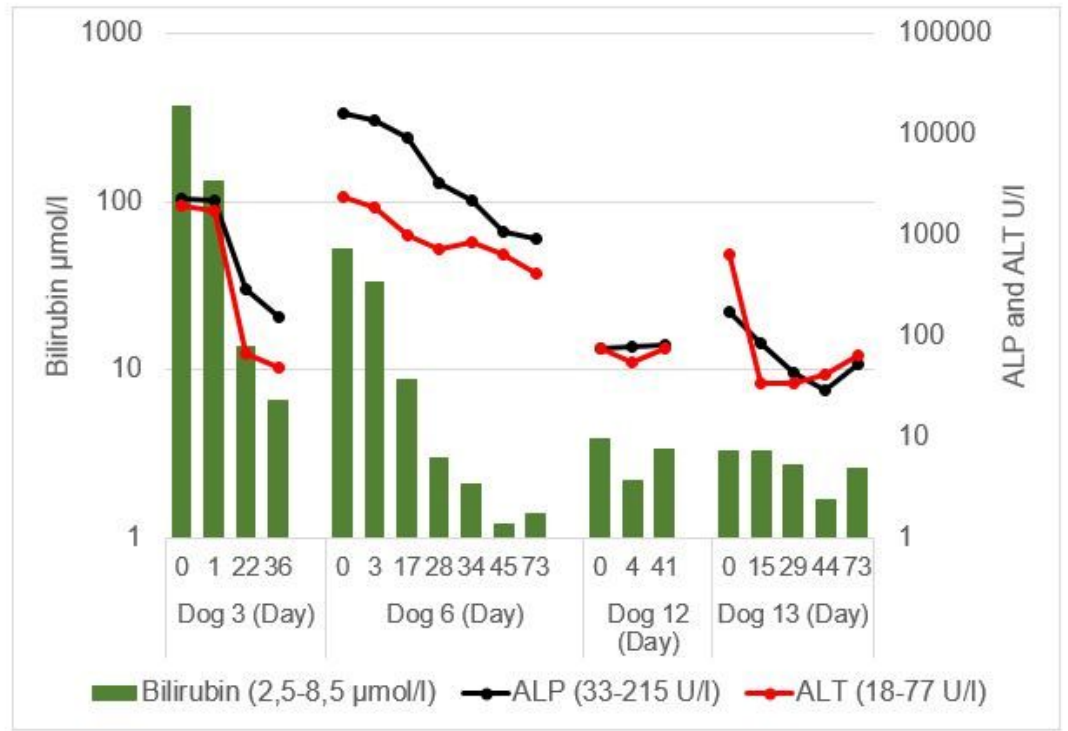

\section{Figure 5}

Serum bilirubin concentrations and serum activities of alkaline phosphatase (ALP) and alanine aminiotransferase (ALT) of four dogs before and after successful EST. 